\title{
Ein Einsatzmodell zur Identifizierung der wesentlichen Einflussgrößen von Portalstaplern auf die Containerterminal- Umschlagleistung
}

\author{
Dipl.-Ing. Loubna Doubli, Prof. Dr.-Ing. Rainer Bruns \\ Helmut-Schmidt-Universität Hamburg \\ Lehrstuhl für Maschinenelemente und Technische Logistik
}

\begin{abstract}
Der Einsatz von Portalstaplern (Van Carrier (VC)) in einem Containerterminal in Seehäfen stellt ein komplexes System dar. Es gibt viele Parameter, seien es Terminalparameter oder Fahrzeugparameter, die die Umschlagleistung eines Portalstaplers beeinflussen können. Um den Einfluss dieser Parameter auf die Umschlagleistung zu ermitteln, wurde ein einfaches mathematisches Modell gebildet, welches dem realen System ähnlich ist. Das Einsatzmodell beinhaltet alle relevanten VC-Arbeitsspiele (Insgesamt 7 Arbeitsspiele für den Containertransport zwischen Schiff, Lager, Bahn- und Lkw-Umschlag).
\end{abstract}

\section{$1 \quad$ Einleitung}

Bauartbedingt neigen Portalstapler bei schneller Kurvenfahrt zum seitlichen Umkippen bevor ein Schleudern des Fahrzeugs auftritt. In Deutschland ereignen sich daher trotz des Einsatzes von Portalstaplern neuester Bauart im statistischen Mittel 0,8 Kippunfälle pro Jahr mit diesen Flurförderzeugen, bei denen der Fahrer meistens schwere oder sogar tödliche Verletzungen erleidet. Auch wenn die absolute Anzahl solcher Unfälle vergleichsweise gering ist, sind wegen der Schwere der Unfallfolgen weitere organisatorische und technische Maßnahmen erforderlich, um den Betrieb von Portalstaplern sicherer zu machen. In größeren Seecontainerterminals kommen bevorzugt 3-hoch- und 4-hoch-Geräte zum Einsatz, was bedeutet, dass die Container in die dritte bzw. vierte Lage gehoben werden können.

Ziel des Forschungsprojektes ist die Erhöhung der dynamischen Standsicherheit von Portalstaplern, ohne die Wirtschaftlichkeit des Einsatzes dieser Geräte zu beeinträchtigen. Deshalb wurde ein realistisches Einsatzmodell zur Identifizierung der wesentlichen Einflussgrößen eines VCs auf die Umschlagleistung erstellt. Das hier beschriebene Berechnungsmodell dient als ein Hilfsmittel bei der Feststellung, ob eine erarbeitete technische Verbesserungsmaßnahme die Wirtschaftlichkeit des Gerätes beeinträchtigen kann. 


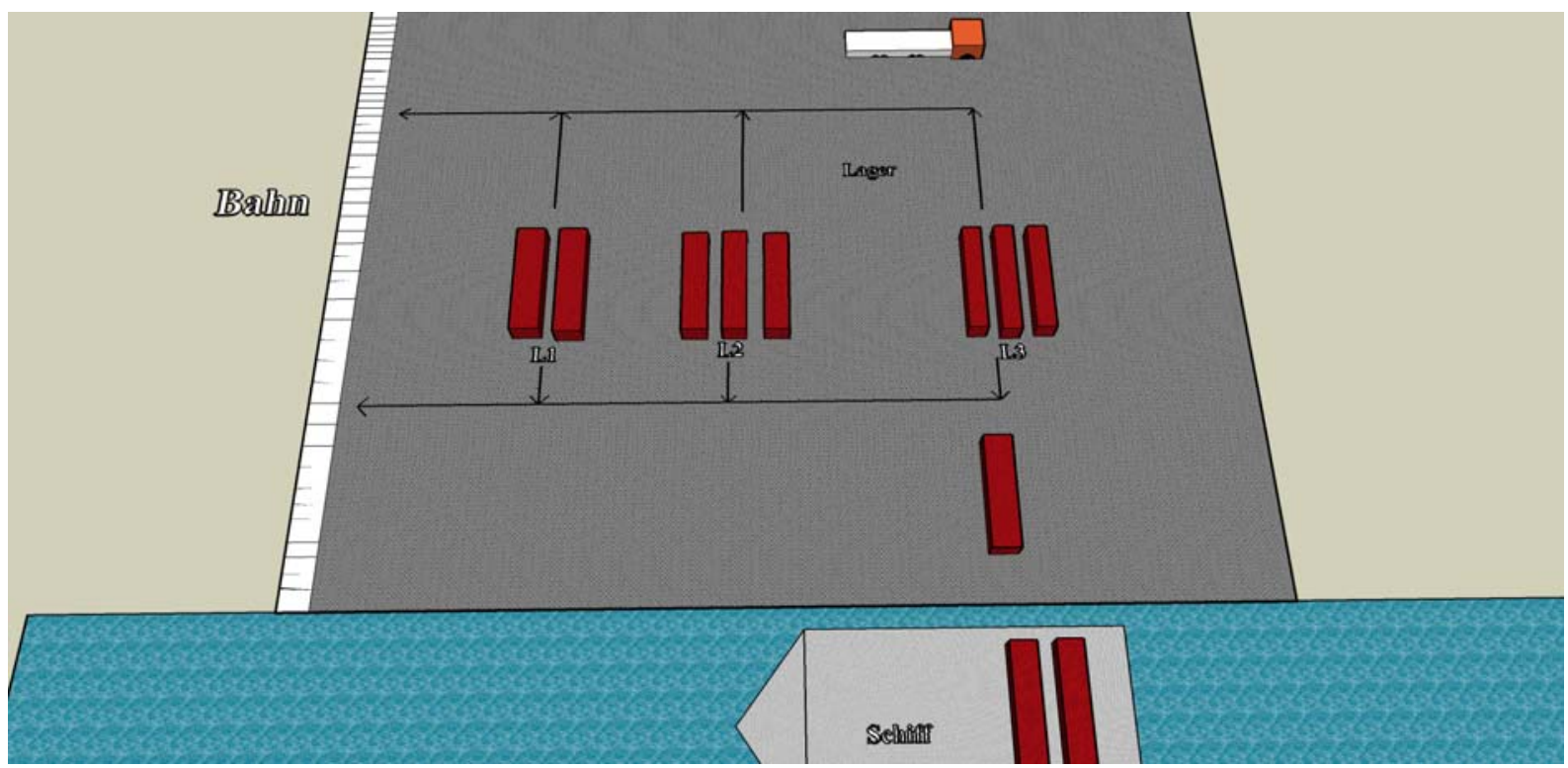

Abbildung 1.1: Schematischer Aufbau eines Containerterminals

\section{Berechnung der Spielzeiten}

Mithilfe der gewonnenen Erkenntnisse aus mehreren Besuchen des ausgewählten Containerterminals und den vor Ort durchgeführten Messungen wurden sieben relevante VC-Arbeitsspiele für den Containertransport zwischen Schiff, Lager, Bahnund Lkw-Umschlag definiert. Bis ein Container mit der Bahn oder dem Lkw weiter transportiert wird, muss er im Blocklager zwischengelagert werden. Deshalb sind die Arbeitsschritte (die letzten Schritte in Abbildung 2.1) im Blocklager bei jedem Auftrag durchzuführen. Die Arbeitsschritte am Kai bei einer Schiffabfertigung, am Lkw oder an der Bahn sind identisch (die ersten Arbeitsschritte in Abbildung 2.1). Daher können diese sieben Arbeitsspiele zusammengefasst und durch das in Abbildung 2.1 dargestellte universelle Arbeitsspiel ersetzt werden. In diesem Abschnitt wird das für die Berechnung der Spielzeit, d.h. der Zeitdauer des universellen Arbeitsspieles, verwendete mathematische Modell beschrieben.

Im Berechnungsmodell für die Zeitdauer eines Auftrages wird zunächst die Zeitdauer einer idealen Fahrt $\Delta t_{\text {Fahrt }}$ (siehe Kapitel 2.1) bestimmt. Die zusätzlichen Zeitver-zögerungen durch Kreuzungen, Kurven, die Stapelung im Blocklager und die Leerfahrt werden durch zusätzliche Terme berücksichtigt. Für die Zeitdauer $\Delta t_{\text {Auftrag }}$ gilt demnach:

$$
\Delta t_{\text {Auftrag }}=\Delta t_{\text {Fahrt }}+k \cdot \Delta t_{\text {Kreuzung }}+n_{e} \cdot \Delta t_{\text {engeKurve }}+n_{w} \cdot \Delta t_{\text {weiteKurve }}+\Delta t_{\text {Block }}+\Delta t_{\text {leereFahrt }}
$$




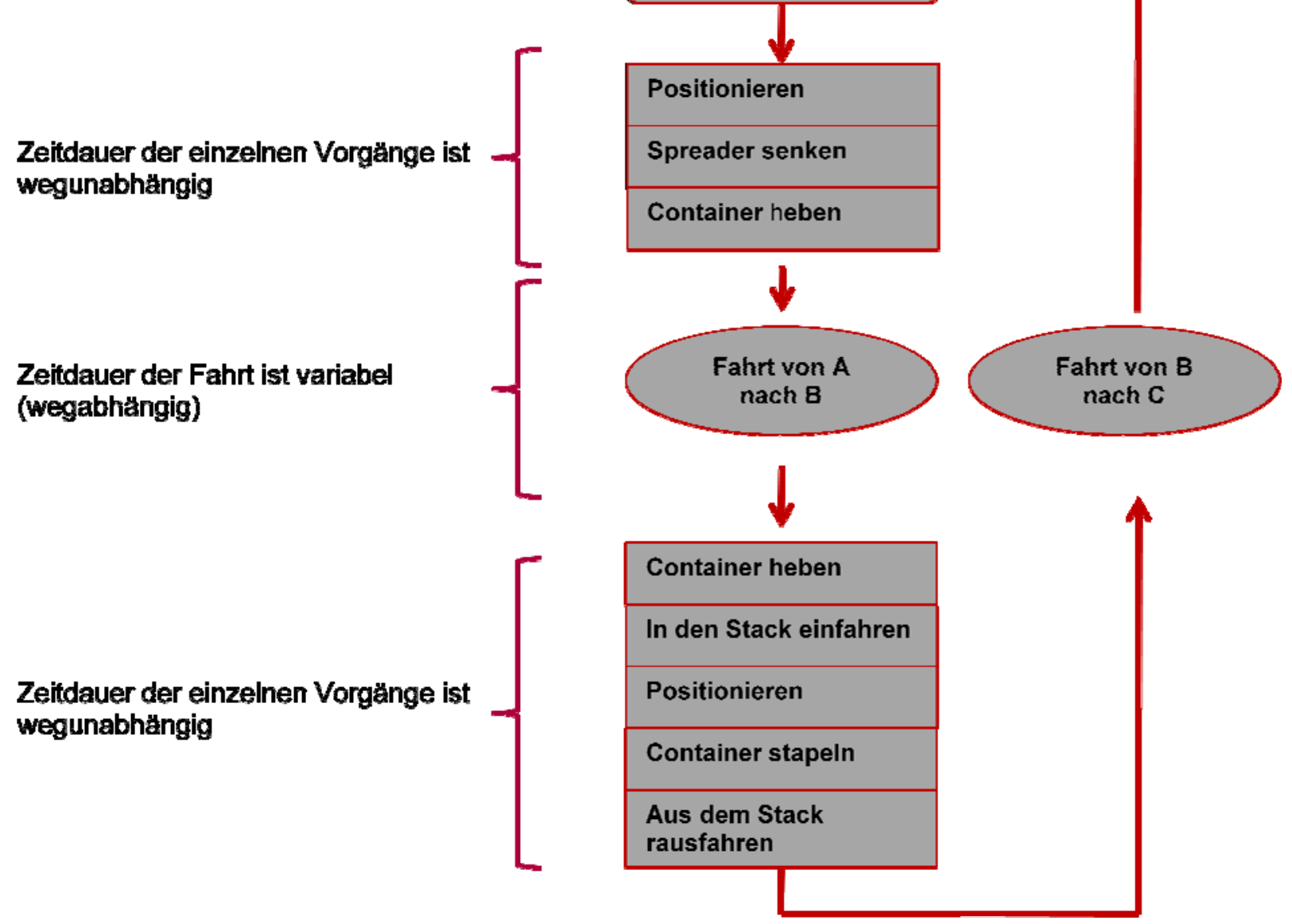

Abbildung 2.1: Arbeitsschritte eines Auftrages

\subsection{Berechnung der Zeitdauer der idealen Fahrt}

Der erste Anteil auf der rechten Seite von Gleichung 2.1 beschreibt die Zeitdauer einer idealen Fahrt. Hierunter ist eine Fahrt über eine vorgegebene Streckenlänge $s$ ohne Kurven, Kreuzungen zu verstehen. Diese wegunabhängige Zeit setzt sich aus der Zeit für die erste Beschleunigung und die letzte Abbremsung sowie aus der Fahrtzeit mit einer konstanten maximalen Geschwindigkeit zusammen, siehe Abbildung 2.2.

Diese Vereinfachung ist möglich, wenn die Beschleunigung und die Abbremsungsstärke konstant bleiben und der Weg $s$ hinreichend groß ist, um die maximale Geschwindigkeit zu erreichen. Unter diesen Voraussetzungen gilt:

$$
\Delta t_{\text {Fahrt }}=\frac{s}{v_{\max }}+\frac{v_{\max }}{2}\left(\frac{1}{a}+\frac{1}{b}\right)
$$




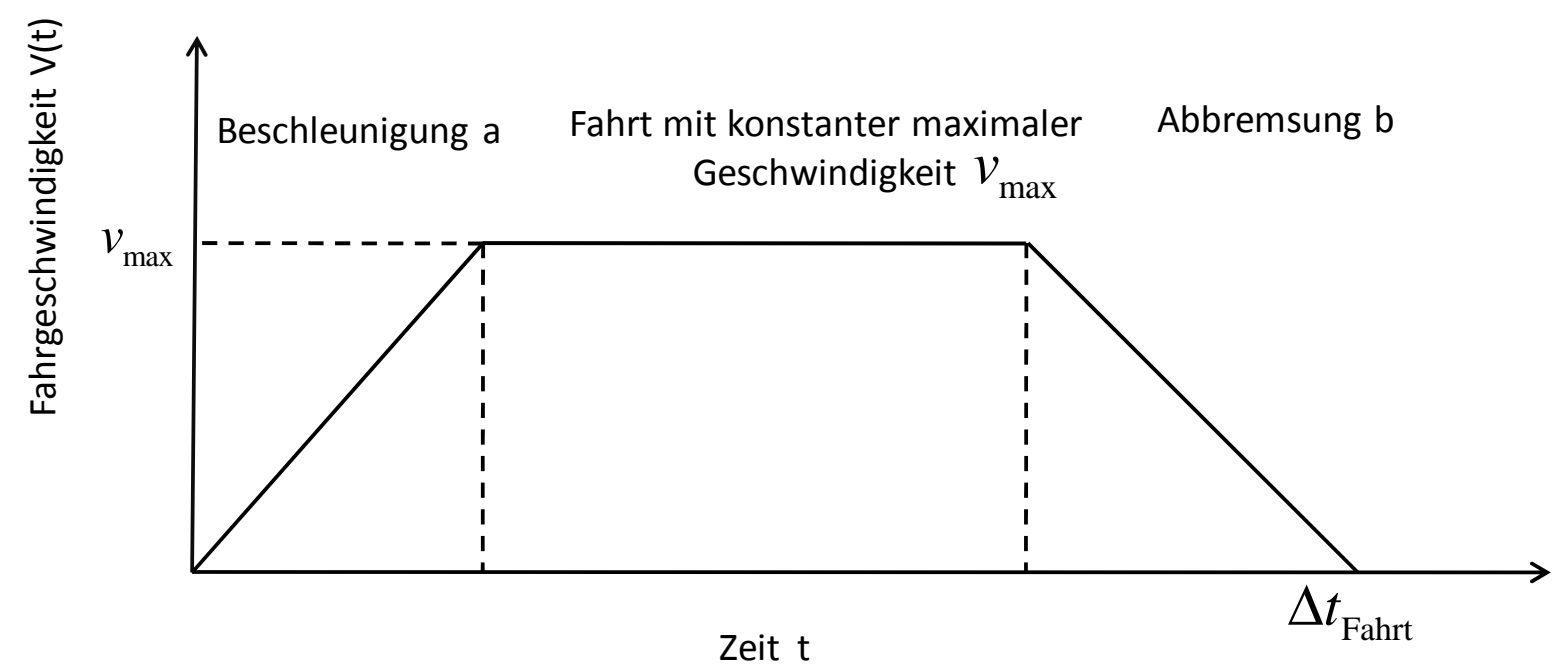

Abbildung 2.2: Idealisierte Fahrt

mit der gesamten Streckenlänge des Auftrages $s$ und der maximalen Geschwindigkeit $v_{\max }$. Sollte die Streckenlänge nicht lang genug sein, um $v_{\max } \mathrm{zu}$ erreichen, d.h.:

$$
v_{\text {max }} \geq v_{\text {grenze }}=\sqrt{\frac{2 \cdot s}{\frac{1}{a}+\frac{1}{b}}}
$$

so gilt für die ideale Fahrtzeit:

$$
\Delta t_{\text {Fahrt }}=\sqrt{2 \cdot s\left(\frac{1}{a}+\frac{1}{b}\right)}
$$

\subsection{Berechnung der Zeitdauer für eine Kreuzung}

Der Faktor $\Delta t_{\text {Kreuzung }}$ in Gleichung 2.1 beschreibt die zusätzliche Zeitdauer durch das Überfahren einer Kreuzung. Mit der Anzahl $k$ der Kreuzungen eines Auftrages ergibt sich hieraus die gesamte Zusatzzeit durch das Überfahren von Kreuzungen für den jeweiligen Auftrag. Die Zeitdauer einer Kreuzung setzt sich aus der Wartezeit (Falls der Fahrer keine Vorfahrt hat) und Fahrzeit der Kurve zusammen. Aufgrund der zwei Kurvenarten sind (siehe Abbildung 2.3) linke bzw. rechte Kreuzungen wie folgt definiert:

$$
\Delta t_{\text {linkeKreuzung }}=\Delta t_{\text {Wartezeit }}+\Delta t_{\text {linkeKurve }}
$$

bzw. 
$\Delta t_{\text {rechteKreuzung }}=\Delta t_{\text {Wartezeit }}+\Delta t_{\text {rechteKurve }}$

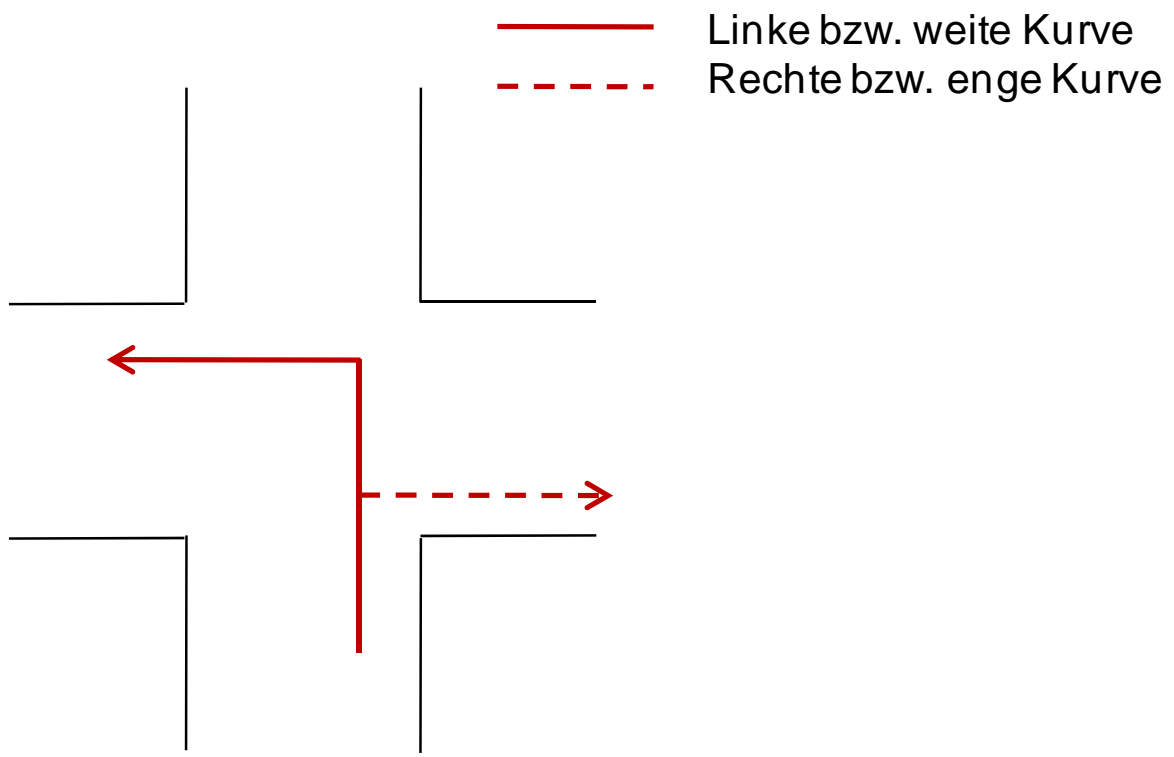

Abbildung 2.3: Kurvenarten

Da die Zusatzzeiten für das Überfahren von Kurven im dritten bzw. vierten Anteil der Gleichung 2.1 separat mit einbezogen werden, braucht an dieser Stelle nur die Wartezeit berücksichtigt zu werden.

Die Zeitdauer einer gerade überfahrenen Kreuzung setzt sich aus der Wartezeit und der Abbremsung- und Beschleunigungszeit zusammen. Die Zeit zum Durchfahren der Kreuzungsstrecke $S_{\text {Kreuzung }}$ wurde bereits als $v_{\text {max }}$-Fahrt in dem Ansatz 2.2 in der Gesamtstrecke $S$ berücksichtigt. Dieser Zeitanteil muss abgezogen werden.

$$
\Delta t_{\text {geradeausKreuzung }}=\Delta t_{\text {Wartezeit }}+\frac{v_{\max }}{a}+\frac{v_{\max }}{b}-\frac{s_{\text {Kreuzung }}}{v_{\max }}
$$

\subsection{Berechnung der Zeitdauer für eine Kurve}

Der dritte bzw. der vierte Term der Gleichung 2.1 beschreibt die zusätzliche Zeitdauer für enge bzw. weite Kurven. Die Zusatzzeit einer Kurve setzt sich aus drei Anteilen zusammen. Der erste Anteil beschreibt die Abbremsungszeit mit der Annahme, dass der Fahrer vor dem Abbremsen die maximale Geschwindigkeit erreicht hat. Der zweite Anteil beschreibt die Phase konstanter Geschwindigkeit in der Kurve. Der letzte Anteil beschreibt die Beschleunigungsphase mit der Annahme, dass der Fahrer die maximale Geschwindigkeit wieder erreicht. Analog zu Gleichung 2.7 wird der $v_{\max }$-Anteil abgezogen. So gilt für die Zeit einer Kurve: 


$$
\Delta t_{\text {Kurve }}=\frac{v_{\max }-v_{\text {Kurve }}}{b}+\frac{\pi \cdot r_{\text {Kurve }}}{2 \cdot v_{\text {Kurve }}}+\frac{v_{\max }-v_{\text {Kurve }}}{a}-\frac{s_{\text {Kurve }}}{v_{\max }}
$$

mit dem Radius der Kurve $r_{\text {Kurve }}$. Die Befragung der Terminalbetreiber und die Messungen vor Ort ergaben, dass der Fahrer keine konstante Geschwindigkeit in der Kurve fährt, weshalb der zweite Anteil der Gleichung 2.8 entfällt.

\subsection{Berechnung der Zeitdauer im Block}

Der fünfte Anteil der Gleichung 2.1 beschreibt die Zeitdauer, die ein Fahrer in einem Lagerblock braucht. Diese Zeit setzt sich aus sechs Zeitanteilen zusammen.

$$
\Delta t_{\text {Block_lager }}=t_{0}+\frac{L}{v_{\text {Block }}}+\Delta t_{\text {Positionieren }}+\Delta t_{\text {Teleskopieren }}+\Delta t_{\text {SpreaderAB }}+\Delta t_{\text {SpreaderAUF }}
$$

Die Zeit $t_{0}$ setzt sich aus der zusätzlichen Zeit für die Abbremsung bis zur Stellposition im Lager und die Beschleunigung von der Stellposition zum Ausgang des Lagers zusammen. Auch in dieser Zeitberechnung muss darauf geachtet werden, ob der Portalstapler im beladenen oder leeren Zustand fährt, da in jedem Zustand ein eigener Wert für die Beschleunigung und Abbremsungsstärke gilt.

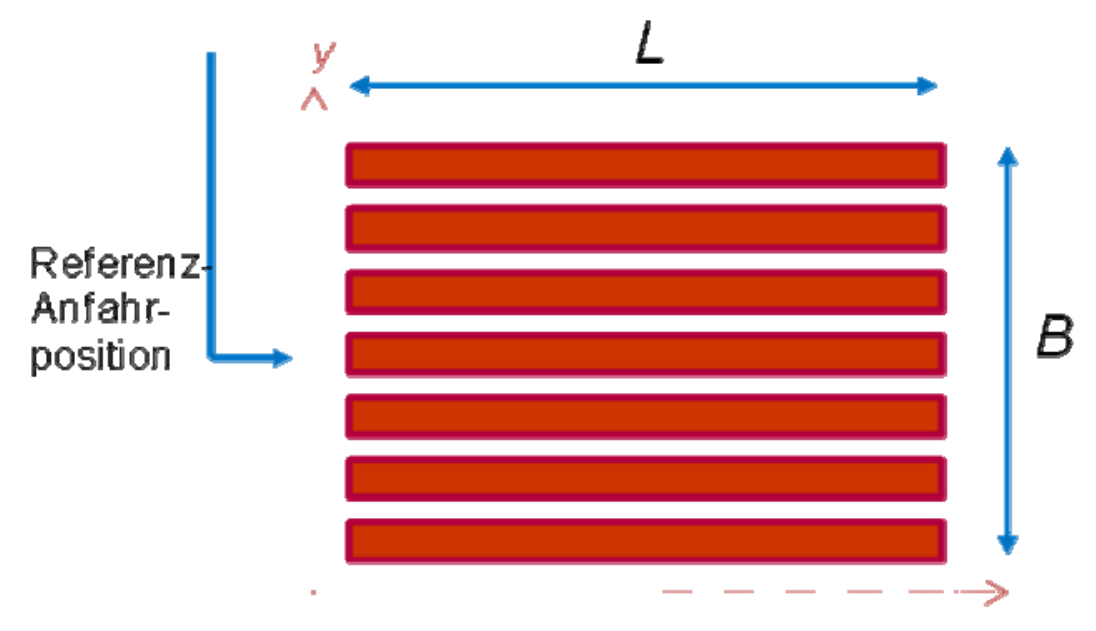

Abbildung 2.4: Lagerblock

Der zweite Term L / $\vee_{\text {Block }}$ ist die Geradeaus-Fahrzeit für die Hin- und Rückfahrt in einem Block der Länge L. $V_{\text {Block }}$ ist die vorgeschriebene Fahrgeschwindigkeit in einem Block. Die mittlere Fahrstrecke ist $L / 2$, so dass für die Hin- und Rückfahrt insgesamt der Weg $L$ zurückgelegt werden muss. Die Länge eines Lagerblocks ist die Länge der Container-Reihen des Blocks. Sind die einzelnen Reihen unterschiedlich lang, so ist $L$ der Mittelwert der einzelnen Reihenlängen.

Zur Vereinfachung des Berechnungsmodells werden die Lagerblöcke des betrachteten Containerterminals in drei Längenklasse (kurzer, mittellanger, langer Lagerblock) unterteilt und zugeordnet. Jede dieser Längenklassen ist durch ein (c) 2010 Logistics Journal : Proceedings - ISSN 2192-9084 
Intervall für den Bereich der Lagerblocklänge und einen repräsentativen Mittelwert charakterisiert.

Die Breite eines Lagerblocks ergibt sich aus der Anzahl der nebeneinander liegenden Containerreihen und deren Breite. Sie hat keinen Einfluss auf die mittlere Fahrzeit, wenn die Referenzanfahrposition für die Berechnung der Weglänge für die Fahrt vom Ausgangspunkt (Schiff, anderer Lagerblock usw.) zum Lagerblock in die Mitte der Seite eines Lagerblocks gelegt wird. Die gefahrene Kurve für das Einfahren in bzw. aus dem Lagerblock wird in der Anzahl der Kurven berücksichtigt.

Umstapelungsvorgänge innerhalb eines Lagerblocks wurden vereinfacht abgebildet. Einzelne Containerpositionen im Lagerblock werden nicht unterschieden. Dadurch werden die Umstapelungsaufträge innerhalb einer Blockreihe ungenau abgebildet.

Die Zeit des Positionierens unterscheidet sich je nach Erfahrung des Fahrers, deshalb wurde bei der Simulation mit einem Erfahrungswert gerechnet. In einigen Fällen muss der Fahrer den Spreader von 20، auf 40، oder umgekehrt teleskopieren. Allerdings teleskopieren die meisten Fahrer während des Fahrens. Der fünfte und sechste Anteil der Gleichung 2.9 beschreiben die Zeit des Aufhebens und Absenkens des Spreaders. Je nach Gerät wird entweder höchstens bis zur dritten Lage (3-hoch Gerät) oder bis zur vierten Lage (4-hoch Gerät) gestapelt.

\subsection{Berechnung der Zeitdauer für eine Leerfahrt}

Der letzte Term der Gleichung 2.1 beschreibt die Zeit der Leerfahrt. Die Leerfahrt ist die Fahrt ohne Container von der Zielposition des letzten Auftrages zu der Startposition des nächsten Auftrages. Diese Zeit beinhaltet im Prinzip die Zeit der idealisierten Fahrt und die Zeitverzögerungen durch die Kreuzungen und Kurven. In der Berechnung wird die Leerfahrt als Auftrag ohne Stapelung im Block betrachtet.

\section{$3 \quad$ Erfassung der Terminalparameter}

Die Bestimmung der Terminalparameter erfolgt anhand des Terminallayouts. Es wird für jede Wegkombination die Anzahl von Kreuzungen, von engen und weiten Kurven und die Größe des Lagers bestimmt. Alle Arbeitsspiele werden betrachtet. Es gibt für den Fahrweg eines Auftrages i.d.R. mehrere Wegvarianten. Der Einfachheit halber werden die Parameter des kürzesten Weges ermittelt.

Die Erfassung aller möglichen Wege ist aufgrund der hohen Anzahl der Wegekombinationen sehr aufwändig. Deshalb wurde die Erfassung im Rahmen des Projektes durch die Benutzeroberfläche CTR (Containerterminal Routing) automatisiert. Bei der CTR Software handelt es sich um ein in Matlab erstelltes Tool, welches zu einem gegebenen Straßennetz den kürzesten Weg zwischen zwei Punkten bestimmt. Weiterhin werden Angaben zu den Gesamtdistanzen, Anzahl und Richtungen der Kurven und Bremspunkten an engen Kurven oder Kreuzungen gemacht. Mit diesen Informationen können VC-Fahrten simuliert und Geschwindigkeitsprofile erstellt werden. 


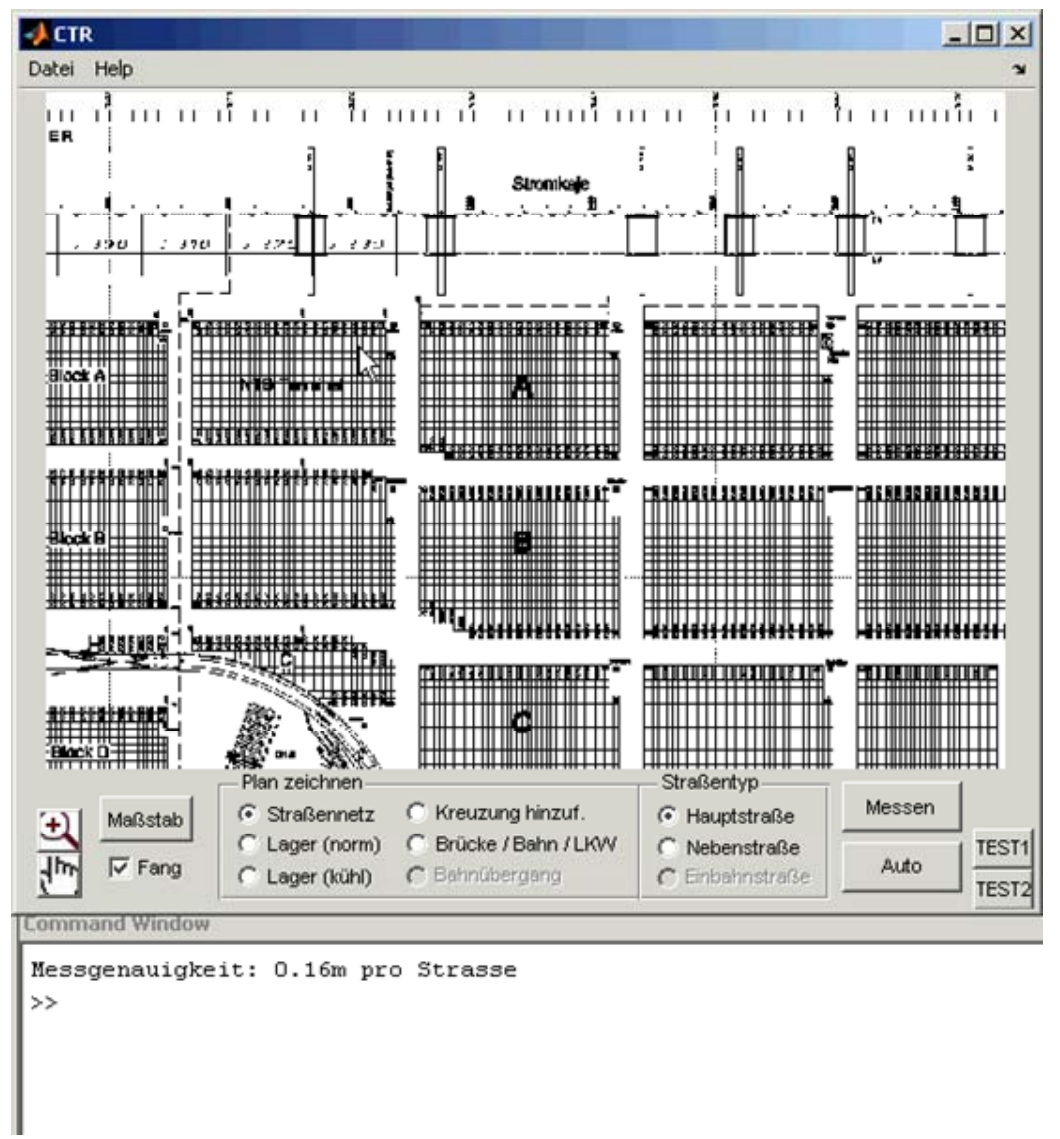

Abbildung 3.1: Benutzeroberfläche CTR

Die Eingabe des Straßennetzes erfolgt ähnlich zu einem Vektor-Grafikprogramm mit der Maus über eine Eingabeoberfläche. In den Hintergrund wird ein Bild der Straßenbzw. Landkarte gelegt, um den Straßenverlauf abzuzeichnen. Hierbei wird zwischen Haupt- und Nebenstraßen unterschieden. Die Straßen werden mit ihren Längen in einer Übergangsmatrix abgelegt. Die passenden Koordinaten $z u$ den Kreuzungsknoten werden in einer weiteren Matrix hinterlegt. Somit kann die Berechnung der kürzesten Wege mit Hilfe des Dijkstra Algorithmus durchgeführt werden.

Um diesen Prozess zu automatisieren, müssen mehrere Start- und Zielknoten bestimmt und benannt werden. Hierfür können den Knoten die Attribute Brücke, Lagereinfahrt, Lagerausfahrt, Kühllager, Bahnlager und LKW-Lager zugewiesen werden. Anhand dieser Informationen wird automatisch der Suchdurchlauf durchgeführt.

Um nach erfolgreicher Pfadfindung ein Geschwindigkeitsprofil erstellen zu können, sind die Distanzen zwischen den Beschleunigungs- und den darauffolgenden Bremsphasen erforderlich. Eine Bremsphase ist als Kurve oder Kreuzung mit Vorfahrtsregelung (rechts vor links) definiert, wobei auf unterschiedliche Geschwindigkeiten abgebremst wird. 
Durch dieses Tool ist es möglich, Straßenverläufe zu aktualisieren und neue Geschwindigkeitsprofile zu erhalten, ohne eine manuelle Neuberechnung der einzelnen Strecken durchzuführen.

\section{$4 \quad$ Validierung des Einsatzmodells}

Für die Durchführung der Simulation wurden reale Auftragslisten verwendet. Zuerst wird aus der Auftragsliste die Zeit ausgelesen, die der Fahrer in der Realität benötigt hat, um den jeweiligen Auftrag durchzuführen. Diese gemessenen Zeiten und die daraus resultierende Umschlagleistung sind die Referenzdaten für die Modellvalidierung.

Zunächst wird für jeden Auftrag der Liste die Spielzeit (wie im Kapitel 2 beschrieben wurde) anhand der Herstellerangaben für die Fahrzeugleistung berechnet. Die Summe aller Spielzeiten wird mit der Referenzleistung verglichen. Dieselbe Berechnung wird anhand der mittleren gemessenen Fahrzeugleistungsdaten erneut durchgeführt. Die Messung der Leistungsdaten wurde mittels zweier Messgeräte durchgeführt. Ein Beschleunigungsaufnehmer zum Messen konstanter Beschleunigungen und ein Datenlogger, der über den CAN-Bus z.B. den aktuellen Lenkwinkel, die Betätigung des Bremspedals, die Geschwindigkeit, die aktuelle Spreaderhöhe und die Quer- und Längsbeschleunigung aufnimmt.

Die Herstellerangaben und mittleren gemessenen Fahrzeugleistungsdaten sind bis auf die Beschleunigung und Abbremsung identisch. Die Portalstaplertypen 3-hoch bzw. 4-hoch können je nach Hersteller maximal $30 \mathrm{~km} / \mathrm{h}$ bzw. 25km/h schnell fahren. Die maximale Beschleunigung bzw. Abbremsung ist laut Hersteller größer als die mittlere gemessene Beschleunigung bzw. Abbremsung, da die maximalen Werte im normalen Betrieb nicht erreicht werden.

Der Vergleich zeigte, dass das entwickelte Modell für den Einsatz eines 3-hochGerätes mit einem Fehler von unter 3\% pro Auftrag den realen Einsatz des untersuchten Terminals hinreichend gut abbildet, wobei der Fehler bei Zugrundelegung der Herstellerangaben für die Fahrzeugleistung (anstatt der gemessenen mittleren Fahrzeugleistungsdaten) deutlich höher ist (der Fehler liegt unter $17 \%$ pro Auftrag).

Für 4-hoch-Geräte wird eine vergleichbare Modellgüte wie für 3-hoch-Geräte erreicht, sodass der Fehler ebenfalls unter 3\% bleibt. Bei Verwendung der Herstellerangaben für die Fahrzeugleistung liegt der Fehler unter 12\%.

Zusätzlich zu der Berechnung der mittleren Umschlagleistung wurde ein durchschnittlicher Auftrag definiert, um eine Vorstellung von der im Mittel auftretenden Streckenlänge, Kurvenhäufigkeit und Kreuzungshäufigkeit zu bekommen. Die Tabelle 4.1 zeigt die über alle vorhandenen Auftragslisten gemittelte gefahrene Streckenlänge, Kurven und Kreuzungen. 
Tabelle 4.1: Die gemittelte gefahrene Streckenlänge, Kurven, Kreuzungen über alle vorhandenen Auftragslisten

\begin{tabular}{|c|c|c|c|c|}
\hline & Streckenlänge & $\begin{array}{c}\text { Anzahl der } \\
\text { Kreuzungen }\end{array}$ & $\begin{array}{c}\text { Anzahl der engen } \\
\text { Kurven }\end{array}$ & $\begin{array}{c}\text { Anzahl derweiten } \\
\text { Kurven }\end{array}$ \\
\hline Mittelwert & 761 & 2.69 & 1.25 & 1.11 \\
\hline
\end{tabular}

\section{$5 \quad$ Zusammenfassung und Fazit}

Im Rahmen eines Forschungsprojektes, in dem Möglichkeiten zur Erhöhung der Standsicherheit von Portalstaplern untersucht werden, wurde der Einsatz dieser Geräte auf Containerterminals in Seehäfen mathematisch modelliert und in ein Berechnungsprogramm implementiert. Trotz der starken Idealisierung bei der Modellbildung kann mit dem Berechnungstool die mittlere Umschlagleistung für den vorgesehenen Zweck hinreichend genau bestimmt werden. So lagen bei der Modellvalidierung mit Hilfe realer Auftragslisten die Abweichungen in der mittleren Umschlagleistung in einer Größenordnung von max. 3\%. Die Allgemeingültigkeit der bisher gewonnenen Erkenntnisse muss jedoch noch durch Berechnungen für weitere Terminals überprüft werden.

Ziel der Entwicklung dieses Einsatzmodells ist es, den Einfluss der Parameter des Fahrzeugs und des Containerterminals auf die mittlere Umschlagleistung zu quantifizieren. Hierdurch sollen Auswirkungen technischer oder organisatorischer Maßnahmen zur Vermeidung von Kippunfällen auf die Wirtschaftlichkeit des Terminalbetriebs im Vorwege abgeschätzt werden können.

Um grundlegende Erkenntnisse aus weitergehenden Parameterstudien unabhängig von vertraulichen terminalspezifischen Daten gewinnen zu können, soll im nächsten Arbeitsschritt ein fiktives Referenz-Containerterminal erstellt werden. Dieses soll repräsentativ für Containerterminals in deutschen Seehäfen sein. 